\title{
ALGUNAS APORTACIONES DE LA ASOCIACIÓN CASTELLANO- MANCHEGA DE SOCIOLOGÍA AL DISCURSO SOCIOLÓGICO
}

\author{
SOME CONTRIBUTIONS OF THE “ASOCIACIÓN CASTELLANO- \\ MANCHEGA DE SOCIOLOGÍA” TO SOCIOLOGICAL DISCOURSE
}

\author{
Giuliano Tardivo \\ Universidad Rey Juan Carlos. Madrid. España/Spain \\ giuliano.tardivo@urjc.es
}

Recibido/Received: 03/09/2015

Modificado/Modified: 14/09/2015

Aceptado/Acceoted: 29/09/2015

\section{RESUMEN}

La Asociación Castellano-Manchega de Sociología celebra su veinte cumpleaños. Ya es hora de balances y de volver a repasar, aunque de forma rápida y no exhaustiva, las principales aportaciones de esta asociación a la sociología española. Los resultados de este estudio demuestran que la ACMS se ha ocupado de teoría, de investigaciones empíricas, de cuestiones metodológicas, de problemas locales y globales.

\section{PALABRAS CLAVE}

Castilla-La Mancha, teoría, local, global, estructura social, Fuerzas Armadas.

\section{SUMARIO}

1. Introducción. 2. El espacio ocupado por la teoría sociológica. 3. ¿Asociación internacional o local? ¿Qué espacio hay para la realidad social manchega y para los temas globales? 4. Individualismo o Colectivismo: ¿qué perspectiva teórica tiene más espacio en artículos y ponencias de la ACMS? 5. Temáticas especialmente tratadas por la revista: las Fuerzas Armadas. 6. Conclusiones. Bibliografía.

\begin{abstract}
The "Asociación Castellano-Manchega de Sociología" celebrates the twentieth anniversary of its creation. It is time to review the balances and to review, although briefly and not exhaustively, the main contributions of this Association to the Spanish sociology. The results of this study demonstrate that ACMS has dealt with theory, empirical research, methodological issues, and local and global problems.
\end{abstract}

\section{KEYWORDS}

Castilla-La Mancha, theory, local, global, social structure, Armed Forces.

\section{CONTENTS}

1. Introduction. 2. The domain of sociological theory. 3. Association: international or local? What space has the social reality of Castilla-La Mancha and the global issues? 4. Individualism or collectivism: What theoretical perspectives come more frequently in articles and papers of the ACMS? 5. Special topics in the review: the Armed Forces. 6. Conclusions. References. 


\section{INTRODUCCIÓN}

La Asociación Castellano-Manchega de Sociología está de celebración. Nació hace veinte años, cuando la crisis económica y social actual era todavía inimaginable y cuando en el aire castellano-manchego y español todavía se podía percibir una cierta efervescencia colectiva, propia de un país en crecimiento económico e intelectual. A la vez, el proceso de expansión y oficialización de la sociología en España progresaba y se consolidaba (Álvarez Uría y Varela, 2000), así como la consolidación de la sociología periférica, desde Granada a La Coruña, pasando por Toledo (Álvarez-Uría y Varela, 2000: 127). El desarrollo territorial y regional de la sociología y las investigaciones, junto a los informes de ámbito local y regional, son una característica peculiar de la sociología española (Pérez Yruela, 2011: 143).

Estos avances se han parado en lo últimos tiempos, y Castilla La Mancha se sitúa hoy entre las cuatro comunidades españolas más pobres (Fundación FOESSA, 2014: 124), y entre las que más retrocesos han sufrido por la crisis (Fundación FOESSA, 2014: 125). Y la alegría dura poco en la casa del pobre: los sociólogos manchegos han tenido la oportunidad, por consiguiente, de estudiar de cerca una realidad con muchos problemas, que se ha acercado al mundo moderno y que se ha desarrollado tarde. Una sociedad que tenía, y en parte sigue teniendo, características y datos estadísticos propios de una comunidad poco desarrollada, como, entre otros, el número de alumnos por profesores y aulas (Braster, Del Pozo y Del Mar, 2006: 17 y ss.), el elevado empleo rural, una tasa de paro del 28,69\%, cinco puntos superior a la media nacional (EPA, 2015), una tasa de abandono escolar del 33,2\% en 2010 (UNICEF, 2009-2010) y, por último, una tasa de riesgo de pobreza del 28,4\%, contra un 22,2\% nacional (Encuesta Condiciones de Vida, 2014). En la clasificación de las regiones europeas de PIB per cápita en paridad de poder de compra, Castilla-La Mancha ocupa el lugar ${ }^{\circ} 188$, y en claro descenso. En España sólo están en niveles inferiores Andalucía, Melilla y Extremadura (Eurostat, 2015). A su vez, Castilla-La Mancha presenta datos y elementos contradictorios y esperanzadores, como una elevada esperanza de vida, una temprana feminización del profesorado y feministas pioneras en sus campos, como, entre otras, Antonia Roldán Fernández, la primera licenciada en Física de España (Muñoz Sánchez, 2006: 364). En los últimos años se ha reducido el porcentaje de población que no se gradúa en la ESO: 30,3\% en 2009-2010, contra el 33,7\% de 2005-2006 (Fundación Encuentro, 2013: 129), aunque sigue siendo un porcentaje demasiado elevado. Incluso se dedicaban más horas de estudio de promedio respecto al resto de España (Callejo, 2007: 147), si bien los resultados académicos no eran tan buenos como era deseable (Centelles Bolós, 2008: 38), así como que se encuentra un elevado número de museos, superior al de la Comunidad de Madrid (154 contra 124) (Anuario Estadístico de España, 2014: 120), mientras que la producción cultural se reduce notablemente si consideramos el número de libros editados (695 en el año 2013 según el INE). Además, gracias a Cervantes, es una de las comunidades autónomas españolas más nombradas en el mundo, aunque nadie ha encontrado todavía con seguridad absoluta ese mítico lugar sin nombre, que podría ser quizá Villanueva de los Infantes (Parra Luna, 2014: 181 y ss.).

El objetivo del presente artículo es hacer un repaso de la historia de la ACMS y de sus principales actividades académicas y de investigación. Tomaremos en consideración la revista de la asociación, Barataria, los Congresos de la Asociación, que desde 1996 tienen lugar todos los años en "la histórica y bellísima Almagro" (Uña Juárez, 2000: 6) y en los 
que han participado sociólogos de renombre nacional e internacional, entre otros Luis González Seara, Juan Díez Nicolás, etc. Por último, citamos también los Premios Fermín Caballero, que se otorgan año tras año a los mejores ensayos breves de ciencias sociales. Ante la imposibilidad, dada la vastísima producción, de analizar ab ovo usque ad mala todos los acontecimientos y toda la producción intelectual de estos veinte prolíficos años, intentamos en el artículo repasar sólo las que son, a nuestro juicio, las principales cuestiones teóricas y metodológicas tratadas a lo largo de este largo tiempo. Así mismo, para dar a este breve artículo un corte más científico y menos "autocelebrativo", vamos a intentar contestar a las cuestiones, que podrían ejercer la función de preguntas de investigación: ¿qué papel tiene la teoría sociológica en estos artículos y en estas ponencias?, ¿se da más espacio a la investigación empírica o se tiene en cuenta la teoría en igual medida?, ¿qué espacio tiene la sociología internacional en el seno de la revista y de los congresos, tanto a nivel de autores extranjeros como a nivel de temáticas y problemas sociales tratados?, ¿se trata de un espacio reducido como podría suponerse de una asociación de ciencias sociales de origen castellano manchego?, y i la investigación local, centrada en la realidad social, política y económica de Castilla-La Mancha, es relevante?

La principal hipótesis que hemos formulado al principio para llevar a cabo el análisis cualitativo y cuantitativo de los artículos de la revista y de las ponencias de los congresos, se refiere a la reducida presencia de autores y temas que podríamos definir como extranjeros o alejados de los confines de Castilla. Si esta hipótesis no se cumpliera, entonces podríamos afirmar que la ACMS ha entendido, con tiempo y adelanto, que la globalización de la economía y de las culturas interrelaciona todo y convierte por ende lo lejano en cercano. A lo largo de estas líneas intentamos aclarar esta cuestión. Por último, otra pregunta que nos hemos hecho al analizar la revista, sus artículos, y los congresos y sus ponencias concierne la perspectiva teórica de los mismos. ¿Qué perspectiva tiene más espacio: el individualismo, tanto en su versión clásica weberiana como en su versión contemporánea (nueva sociología del individuo), o el colectivismo marxiano y durkheimiano? Dejamos de lado, a sabiendas, un tercer modelo que parece perfilarse en la sociología contemporánea: el llamado modelo de flujos, que se diferencia notablemente del atomismo individualista como del estructuralismo sistémico, pero que está todavía in fieri (García Selgas, 2015: 77). Por ejemplo ¿el concepto de clase social tiene mucho espacio en Barataria o ya se prefigura su crisis como operador analítico? ¿Hay alguna temática que aparezca a menudo en los artículos y en las investigaciones y que la ACMS quiera ampliar de especial manera?

Utilizamos la metodología clásica del análisis de documentos, sobre todo de tipo cualitativo (Perelló Oliver, 2009), para llevar a cabo la reflexión y el balance sobre esta asociación de sociología y su legado, así como el análisis crítico del discurso, que se inspira en el Critical discourse analysis de Norman Fairclough (Mantovani, 2008: 17), para relacionar los discursos de los miembros de la ACMS con categorías y operadores analíticos más amplios y "para indagar el funcionamiento de ámbitos de relaciones sociales y de las categorías (...)" (Martín Criado, 2014: 117). Advertimos de que como marco conceptual y teórico de referencia tendremos en consideración Castilla-La Mancha y a la vez la sociología española e internacional, su rumbo actual y su evolución en las últimas décadas. Estas reflexiones que llevamos a cabo a continuación nos servirán para comprobar si la sociología manchega converge o diverge de la sociología española e internacional. 


\section{EL ESPACIO OCUPADO POR LA TEORÍA SOCIOLÓGICA}

¿Cuántos son los artículos de teoría sociológica publicados en Barataria y de qué se ocupan? En el primer número de la revista Barataria su entonces director, y fundador de la ACMS, el profesor Octavio Uña, dedica un artículo a la teoría sociológica. En el segundotercer número hay al menos un artículo que puede ser considerado de teoría sociológica, dedicado al pensador y sociólogo Julien Freund (Molina, 2000: 177 y ss.). El autor quiere dar a conocer el pensamiento sociológico de este científico social, poco estudiado en España. En el quinto número de la revista encontramos otros dos artículos que podemos considerar de teoría sociológica (Martín Cabello, 2002; Garrigós Monerris, 2002). En el séptimo número de Barataria el profesor Octavio Uña (2005-2006: 91 y ss.) presenta un recorrido de los principales pensadores de la sociología académica. En el octavo número de Barataria, la profesora de la URJC, Almudena García Manso (2007: 13 y ss.), reflexiona desde un punto de vista teórico sobre el ciberfeminismo, su origen y su evolución. La teoría, en este caso, sirve también para mirar al futuro. En el undécimo número de Barataria, el profesor y periodista Maximiliano Fernández Fernández (2010: 141) recuerda la contraposición histórica entre la sociología europea, más teórica, y la norteamericana, más cuantitativista y empírica. Se trata de una contraposición que ha dejado de tener sentido hoy día. Ambas posturas, si se toman de forma radical y sectaria, pueden dar lugar a sendas a aberraciones científicas. En el decimocuarto número encontramos otro artículo teórico escrito por el profesor Pedro García Bilbao, profesor de sociología y miembro histórico de la ACMS. Tratando el tema de las migraciones forzosas, García Bilbao (2012: 158) llega a afirmar que Plutarco es "un precursor de la mirada sociológica". Y el profesor Jaime Hormigos (2012: 75 y ss.) presenta las principales reflexiones teóricas de los grandes de la sociología sobre la música como hecho social. En el decimosexto número de la revista Barataria tenemos al menos un artículo de teoría sociológica, escrito por el profesor Urteaga (2013: 15-31), y centrado en la figura de Norbert Elias recogiendo la polémica en torno a su figura y a las dificultades que han acompañado el proceso de reconocimiento y aceptación de éste como uno de los grandes sociólogos, a la vez que Weber, Durkheim, Pareto, etc. En el mismo número el profesor de psicología de la Universidad de A Coruña, Miguel Clemente, junto con otros colaboradores, presenta los resultados de una investigación empírica realizada sobre los inmigrantes chinos, una investigación que no rechaza la teoría y se apoya en una sólida base teórica y conceptual, como demuestra la reflexión que los autores llevan a cabo sobre el concepto de diáspora (Clemente et al. 203: 83 y ss.).

En el número 18 de Barataria, Roberto Barbeito y Ángel Iglesias (2014: 215 y ss.) ponen en evidencia los límites del actual concepto de democracia y las posibles mejoras que se pueden implementar para aumentar la participación ciudadana.

En los congresos de la ACMS el panorama es parecido y se entremezclan ponencias de teoría sociológica con otras centradas en los resultados de investigaciones más prácticas. En el XII Congreso destacamos, por ser el primero del que se conservan las actas, en cuanto a teoría sociológica, entre otras, las ponencias de Octavio Uña titulada "Reflexiones sobre la sociología como ciencia" (Uña Juárez, 2007: 133-158), igualmente la de Eduardo Díaz Cano centrada en la figura de Ernst Troeltsch, autor apenas conocido en España, y que el mismo autor se ocupó de sacar de las tinieblas y del desconocimiento en su tesis doctoral (Díaz Cano, 2007: 27-42). Troeltsch merece ser reconocido como uno de los primeros sociólogos que intuyó el proceso de trasformación de la religión en algo más que un tema "personal e interno" (Beriain, 2015: 16). En el XIII Congreso sobre técnicas de 
investigación de Ángel José Olaz Capitán (ACMS, 2008: 145-155); en el XIV Congreso sobre el pensamiento organizativo de José M. Cortés Martí (ACMS, 2009: 395-408), sobre la globalización de Francisco Entrena Durán (ACMS, 2009: 433-454) o el conflicto de Jaime Hormigos Ruiz (ACMS, 2009:469-486); en el XV Congreso, sobre cultura de Antonio Martín Cabello ACMS, 2010:235-252) o redes sociales de Antonio García Benítez (ACMS, 2010:275-286) y Sara Gallego Trijueque ACMS, 2010:298-306);

Podemos afirmar, pues, que en Barataria y en la ACMS la sociología no parece ni un inútil ejercicio retórico, ni un simple análisis empírico de lo inmediato. Tanto fundadores como colaboradores de la ACMS parecen haber encontrado el mesotes aristotélico que otras revistas y otras asociaciones parecen haber perdido con su énfasis excesivo en los datos y en el empirismo cientifista. Estas revistas parecen seguir al pie de la letra la creencia o el dogma de que "el paso de lo cualitativo a lo cuantitativo (...) siempre ha supuesto un progreso" (López López y Escalda López, 1999: 162).

Se trata de algo que se hace explícito también repasando los ensayos galardonados por la ACMS con el Premio Fermín Caballero. El IX premio lo obtuvo De la Calle Valverde (2011: 10), un autor que polemiza abiertamente con las "constricciones a los que el mundo científico somete hoy" a los investigadores. En el seno de la ACMS es posible todavía, sin embargo, elaborar un pensamiento crítico o pronunciar palabras prohibidas en otros lugares de la sociología española, como por ejemplo: "pues, en mi opinión..." (De La Calle Valverde, 2011: 29). Un riesgo, el de la excesiva positivización y cuantificación de la sociología española, compartido incluso por autores no contrarios a la modernización e internacionalización de la disciplina: "si sociología acaba siendo -dice Pérez Yruela (2011: 145)- sinónimo de encuesta, por muy importante que sean, podemos estar haciendo una sociología técnicamente impecable pero sociológicamente poco relevante". Por otro lado, la ACMS ha conseguido librarse del defecto opuesto, "el ensimismamiento" o "filosofización" de la sociología (Noya, 2004: 166). A este respecto, en el artículo del profesor Uña citado a principios de párrafo, el catedrático de sociología de la URJC reconoce la importancia de las teorías de alcance medio en la sociología (Uña Juárez, 1998: 17), como punto de equilibrio entre los dos extremos opuestos.

Tabla 1: Artículos dedicados a la teoría sociológica en Bar
\begin{tabular}{|l|}
\hline Uña Juárez, $1998, \mathrm{n}^{\circ} 1$ \\
\hline Martín Cabello, $2002, \mathrm{n}^{\circ} 5$ \\
\hline García Manso, $2007, \mathrm{n}^{\circ} 8$ \\
\hline Pascal, 2010, $\mathrm{n}^{\circ} 11$ \\
\hline Fernández Fernández, 2010, $\mathrm{n}^{\circ} 11$ \\
\hline Hormigos Ruíz, $2012, \mathrm{n}^{\circ} 14$ \\
\hline García Bilbao, $2012, \mathrm{n}^{\circ} 14$ \\
\hline Urteaga 2013, $\mathrm{n}^{\circ} 17$ \\
\hline
\end{tabular}

Fuente: elaboración propia

En Barataria y al mismo tiempo en todas las actividades organizadas por la ACMS, se hace evidente que los científicos sociales manchegos han entendido con adelanto la necesidad de abrir un diálogo nuevo y continuo entre historia, economía, sociología y las demás ciencias sociales (Camas Baena, 2014: 150), porque constituyen "una única aventura del espíritu", como diría Braudel. Jon Elster a este respecto afirma que "ya es hora de construir un nuevo paradigma para las ciencias sociales" (Perulli, 2015: 11-115). 


\section{ASOCIACIÓN INTERNACIONAL O LOCAL. ¿QUÉ ESPACIO HAY PARA LA REALIDAD SOCIAL MANCHEGA Y PARA LOS TEMAS GLOBALES?}

La colaboración de la ACMS con el Colegio de la Frontera Norte de Tijuana, Méjico, con la Universidad de El Cairo y otras universidades demuestra la aspiración internacional y no sólo local de la ACMS. La colaboración con profesores de otros países, como K. Gamal, Jorge Santibáñez, Peadar Kirby o Bernard Labatut, entre otros, añade elementos positivos en relación a la perspectiva internacional. Además, algunos miembros históricos de la Asociación, coordinados por los profesores Uña Juárez, Hormigos Ruiz y Martín Cabello (2007), publicaron un libro colectivo sobre la globalización, lo cual prefigura la apertura de la Asociación a los temas globales, más allá de los confines manchegos. Vamos a ver ahora si más allá de la colaboración con universidades y profesores, también en la revista Barataria se respira este aire internacional.

Por ejemplo, el artículo de Molina sobre Freund dedica varias páginas a la polémica entre irenismo y polemología, un tema internacional o global (Molina, 2000). En el quinto número un artículo sobre tecnologías de Internet abre las puertas al mundo contemporáneo. En el undécimo número interviene una autora extranjera, Celine-Marie Pascal (2010: 3139) de la American University Washington. En el decimotercero número de Barataria Suárez Ávila (2012: 195 y ss.) trata el caso de la política migratoria en San Francisco, un tema que abre los intereses de la ACMS al mundo. En el decimoséptimo número ocurre algo parecido, con el artículo de Pérez Fuentes (2014: 97 y ss.) sobre los niños migrantes en México y con el artículo de Andaluz Vegacenteno (2014: 177 y ss.) sobre los derechos constitucionales en Bolivia.

Sin embargo, no faltan artículos que se dedican al tema castellano manchego, así como las investigaciones centradas en profundizar sobre la realidad y la actualidad de esta comunidad. Desde este punto de vista, nos gusta recordar los trabajos de investigación llevados a cabo por Octavio Uña y Felipe Centelles -quien ya no está entre nosotros- pero que dedicó sus mayores esfuerzos intelectuales en dar a conocer la realidad manchega como lo atestiguan sus variadas obras al respecto. Centelles, por ejemplo, investigó la percepción que tienen los jóvenes universitarios manchegos a propósito de la identidad manchega y subrayó, en el seno de la misma, los avances que se han producido en los últimos años señalando el hecho de que se crease una Universidad de Castilla-La Mancha (Centelles Bolós, 2008: 33-37). Entre otras investigaciones tenemos que recordar la que realizó junto a otros profesores sobre la construcción de la identidad política en Castilla-La Mancha. Una investigación llevada a cabo en una época en la que el bipartidismo PPPSOE parecía el único panorama político posible en esta comunidad. Lo autores presentan la evolución política y social de Castilla-La Mancha y la difícil construcción de la identidad regional, respecto a otras comunidades que tienen lengua propia y una subcultura específica (Bleda García, Centelles Bolós y Mora, 2000: 27). Los investigadores de la ACMS explican, en ese mismo trabajo, también las razones de la ausencia de una verdadera identidad regionalista en Castilla-La Mancha, frente a una fuerte identidad localista o municipalista y a la vez españolista. Se trata de un tema que ha sido retomado por otros autores. Sánchez Rodríguez y Punzón Moraleda (ACMS, 2007: 579 y ss.) en el XII congreso de la ACMS pusieron en evidencia el papel ejercido en la construcción de la identidad manchega por el liderazgo carismático de José Bono. El profesor Uña (2008) volvió a tratar este tema en una breve presentación que escribió para introducir un volumen sobre los 25 años de autonomía castellano-manchega. 
El mismo investigador de la ACMS, José María Bleda (2002), realizó una investigación sobre el Estado del Bienestar en Castilla-La Mancha que, entre otros aspectos positivos, confirma, sin embargo, que los sociólogos raramente aciertan con las previsiones de futuro. Este estudio fue realizado a principios del siglo XXI, cuando los recortes y la crisis todavía no se podían ni intuir ni imaginar. El Estado del Bienestar continental, mediterráneo, manchego, que otros denominan como "welfare bismarkiano" (Ingellis y Calvo, 2015: 11), como todos los que se inscriben dentro de este modelo, ha mostrado en los últimos tiempo carencias y debilidades, como el llamado efecto Mateo, más que "ampliar y mejorar la situación actual" como preconizaban los expertos entrevistados en aquellas fechas (2002: 9). Y continuando con los entrevistados, el 89,2\% preveía una mejora de la asistencia sanitaria para los inmigrantes en el periodo 2000-2015. Se trata, como es bien sabido, de uno de los colectivos más afectados por los recortes. Sí acierta en sus afirmaciones al recoger en su artículo que "en el futuro las administraciones públicas no van a tener el monopolio de la responsabilidad de las políticas sociales" (Bleda García, 2002: 115). Continuando con la misma investigación (2002:119), se hacen otras dos afirmaciones en las que los expertos una vez aciertan, y es la que hace referencia al papel de las ONG, de Cáritas y de otros organismos que es hoy reconocido y apreciado por todos, pero en su segunda previsión la dura realidad de la crisis ha demostrado que las desigualdades, lejos de disminuir en el acceso al sistema educativo, previsto por el $48 \%$ de los encuestados han aumentado.

Otra investigación llevada a cabo por el mismo equipo investigador de la ACMS se ha centrado en la mujer castellano-manchega (Uña Juárez, Bleda García y Centelles Bolós, 1999). Un estudio que no se limita a profundizar sobre las mujeres, sino que abarca cuestiones como el cambio demográfico, los movimientos migratorios, el trabajo, etc. El mismo equipo investigador llevó a cabo un estudio más específico sobre la mujer viuda en Castilla-La Mancha. Asimismo, Martínez Andrés y De los Cobos Arteaga (ACMS, 2009: 567 y ss.) demuestran con su intervención que la ACMS está abierta a temas de carácter político y de ciencias de la administración. Los autores en este caso afrontan la cuestión de la pérdida de soberanía de los gobiernos nacionales y del alejamiento entre políticos y ciudadanos, dos temas propios de nuestra era hiperglobalizada y neoliberal (ACMS, 2009: 569). Los autores presentan de forma crítica los principios fundamentales de la nueva gestión pública.

El octavo número de Barataria recoge las consideraciones del profesor Peadar Kirby sobre estrategias de desarrollo y reducción de la pobreza en Irlanda; pero a la vez está siempre presente la perspectiva local, como demuestra el artículo sobre el uso que hacen del tiempo los castellanos-manchegos (Callejo, 2007: 143 y ss.). En el décimo número de Barataria se cumple este doble reto, esta perspectiva local e internacional a la vez, con la participación del profesor italiano, Vincenzo Romania (2009: 39 y ss.), experto en interaccionismo simbólico e inmigración. Y, también, con el análisis del fenómeno inmigratorio en Castilla-La Mancha, llevado a cabo por Antidio Martínez de Lizarrondo Artola (2009: 75 y ss.).

En el decimotercer número de la revista, un artículo que tiene un enfoque claramente global o internacional es el de Benito Gil (2012: 31 y ss.). El autor, entre otras cuestiones, analiza los problemas de convivencia entre inmigrantes y autóctonos, en progresivo aumento desde el inicio de la crisis económica. Las estadísticas, en realidad, demuestran que los inmigrantes han jugado un papel positivo en España, favoreciendo la emancipación de la mujer española y su inserción en el mercado laboral (Fundación 1 de mayo, 2013: 13). 
En el decimoctavo número de Barataria (Mañas Ramírez y García Maestro, 2014) se presentan también los resultados de una investigación cualitativa, llevada a cabo por sociólogos y miembros del personal sanitario.

En el XII Congreso de Almagro tenemos tanto ponencias centradas en temáticas locales como las de Garrido García (ACMS, 2007: 529 y ss.) y García González (CMS, 2007: 569 y ss.). Este último explica el aumento de la longevidad en Castilla-La Mancha, un fenómeno especialmente relevante en esta comunidad, así como investiga sobre la disminución de la fertilidad, un hecho social sólo parcialmente suavizado por la fuerte inmigración de los últimos quince años (García González, 2007: 572). Tampoco faltan, en el seno del mismo congreso, ponencias relacionadas con la globalización y con temas de carácter global o internacional.

En el XIII Congreso, Trinidad Noguera Gracia (ACMS, 2008: 416) demuestra que la ACMS está abierta a temas no sólo de carácter internacional, sino también de historia contemporánea no española, al hablar de la Primavera de Praga y del 68 Francés. La autora relaciona la modernidad líquida de Bauman con el crisol de movimientos que protagonizaron aquellos acontecimientos históricos. En el XIV Congreso ocurre algo parecido: la perspectiva local manchega es objeto de la atención de Simón Medina y Olaya (ACMS, 2009: 499 y ss.). Además, el Congreso se abre a otros países como Francia y a temáticas globales como las migraciones.

\section{INDIVIDUALISMO O COLECTIVISMO: ¿QUÉ PERSPECTIVA TIENE MÁS ESPACIO EN ARTÍCULOS Y PONENCIAS DE LA ACMS?}

El profesor Uña en el primer número de Barataria deja claro desde el principio que individuo y sociedad se influyen mutuamente y que es hora de superar esta contraposición (Uña Juárez, 1998: 11), y recuerda la idea de Luhmann quien afirmaba que individuo y sociedad son dos realidades interdependientes (Uña Juárez, 1998: 19). Por último, sostiene que las bases del colectivismo durkheimiano se encuentran en la idea de los hechos sociales, que se imponen a los individuos (Uña Juárez, 1998: 22). El mismo profesor Uña (2005-2006: 93) en otro artículo de Barataria afirma que en Durkheim "la sociedad es el origen de la verdad", pero a la vez recuerda que el condicionamiento social y las representaciones colectivas en Durkheim son conceptos ambivalentes. En otro texto el catedrático de la URJC vuelve a sostener que conocimiento y acción, teoría y empiria, son dos caras de una misma realidad (Uña Juárez, 2008: 14).

Según Molina (2000: 182), la recepción del pensamiento de Weber en España ha sido temprana, al menos respecto a la sociología anglosajona. En el segundo-tercer número Molina (2000) habla de Freund, quien, entre otras cosas, hizo conocer en Francia la polémica de Weber contra la metafísica y "contra la ideologización de la sociología" (Molina, 2000: 193). Es sabido que Weber consideraba a los individuos como principal unidad de análisis de los sociólogos (Uña Juárez, 1998: 23), aunque el individualismo hermenéutico-weberiano tiene sus raíces lejanas en Aristóteles (García Selgas, 2015: 71). Aunque metodólogos como Noguera ponen en duda la pertenencia acrítica de Weber a esta perspectiva ontológica. Según Noguera la contraposición acción-estructura no equivaldría a la contraposición entre individuo y sociedad (Noguera, 2003: 103) y el mismo Weber utilizaba a veces conceptos supraindividuales para explicar la realidad social (Noguera, 2003: 123). 
El artículo de Martín Cabello, profesor de la URJC, sobre la sociología de la cultura, toca indirectamente esta temática de carácter teórico y pone en evidencia que el concepto de estructura ha sido utilizado por los sociólogos de la cultura en sus análisis mucho más que por los antropólogos (Martín Cabello, 2002: 175).

En el artículo que Urteaga dedica a Elias, se pone en evidencia la influencia ejercida sobre Elias por el individualismo y el racionalismo weberiano. Elias, dice Urteaga (2013: 18), rechaza claramente la idea marxiana que las clases sociales son los únicos operadores analíticos válidos para describir una sociedad. Urteaga (2013: 24) recuerda que al fin y al cabo Elias rechazaba la contraposición entre individualismo y holismo. El individualismo es culpable, según Elias, de centrarse sólo en un aspecto del hombre, sin tener en cuenta el "carácter multidimensional" de la personalidad humana (Uriarte, 2013: 25). La metáfora de la "carrera de antorchas de las generaciones", que el mismo Elias elaboró, pone de relieve que los grupos presentes y pasados influyen sobre el individuo, y a la vez que los sujetos no son receptores pasivos (Vera, 2012: 245). El profesor García Bilbao (2012: 169) nos recuerda que el conflicto entre lo individual y lo colectivo es esencial incluso en el tema de las migraciones forzosas, y que ya los antiguos griegos supieron armonizar la identidad con lo universal. Vincenzo Romania (2009: 54), en su artículo sobre los mediadores culturales en Padua (Italia), concluye su análisis con una interesante reflexión de carácter epistemológico: "es posible estudiar fenómenos macrosociales a partir de una perspectiva interaccionista". Afirmación esta última que confirma la inutilidad de contraponer individuo y sociedad, micro y macro, y que se relaciona claramente con la nueva sociología del individuo, que quiere estudiar lo macro a partir de los individuos, y que implica la idea "de ida y vuelta entre micro y macro" (Noguera, 2003: 112). Una perspectiva que tiene cierto espacio en la sociología francesa contemporánea y que se aleja de la vieja sociología del individuo de Collins (Santiago García 2015), quien, por su parte, consideraba los fenómenos macro como suma de fenómenos micro (García Selgas, 2015: 72).

En los XII premios Fermín Caballero el primer trabajo premiado es un estudio sobre los jóvenes, una "categoría zombi" según algunos sociólogos, o bulldozer, por ser demasiado genérica, pero que, sin embargo, se sigue utilizando mucho en los estudios sociológicos. En realidad, la autora (Cano Hila, 2014: 11 y ss.) critica la idea de los jóvenes como seres iguales, todos vagos y sin ganas de trabajar, como parece venderse en los medios de masas y cómo piensan también algunos profesionales del sector educativo, que a veces utilizan los pocos casos a su alcance y los convierten en modelos metonímicos de todos los jóvenes españoles (Martín Criado, 1998: 35).

De la Calle Valverde (2011: 9 y ss.), que recibió el IX premio Fermín Caballero por un artículo sobre las tesis doctorales, pone en evidencia cómo los ritos de paso han perdido espacio en nuestra sociedad y las estructuras han sido sustituidas por un mayor individualismo. Se trata de algo que se puede percibir en todos los ámbitos de la vida, incluido el religioso, un campo en el que "todas las religiones (...) preaxiales, axiales y postaxiales, se muestran disponibles de apropiación individual en todo momento y en todo lugar" (Beriain, 2015: 13). De Miguel Calvo (2011: 49 ss.), que recibió el mismo año el tercer premio, nos revela que las emociones tienen un claro componente social, y no sólo individual como se ha venido afirmando desde la psicología. El mismo concepto de estilo de vida, a pesar de los diferentes intentos de conceptualización, siempre tiene una cierta referencia al individuo y a la vez a la estructura social (Dumont y Clua García, 2015: 86). En el XII Congreso de 2008, Noguera Gracia (ACMS, 2008: 422) recuerda que vivimos en 
la época de la muerte de los grandes relatos omnicomprensivos y absolutos, como el socialismo, y que se ha ido consolidando en nuestras sociedades el pensamiento débil. Entre grandes estructuras e individuos, los segundos parecen prevalecer. También la sociología actual, y no sólo la ideología política, está atravesando por la crisis de los "cuerpos teóricos con vocación totalizadora" (Pérez Yruela, 2011: 136). De hecho, simplificando cuestiones que necesitarían trabajos más específicos, podemos afirmar que la crisis de las utopías del siglo XIX y XX y de los grandes relatos (González-Anleo, 2014: 105) coincide con la crisis del holismo, y de todas las perspectivas teóricas colectivistas (Noguera, 2003: 121). Roberto Barbeito, en el seno del mismo congreso (ACMS, 2008: 159) recuerda las críticas a Durkheim por su planteamiento epistemológico estático y macrosociológico, que no tiene en cuenta los factores no estructurales de la vida social.

Si las clases sociales no encuentran mucho espacio en los artículos de Barataria y ya se prefigura la crisis de este concepto como operador analítico, lo cual confirmaría indirectamente la idea de que en la sociología actual las categorías analíticas no pueden sustituir a la observación (Spreafico, 2015: 50) sí que se habla sin embargo en la revista de partidos políticos. Por ejemplo, en el decimoséptimo número, en un artículo de López Álvarez (2014: 203 y ss.). El autor subraya las diferencias en el debate político español, respecto a otros países del entorno europeo y a propósito del debate sobre la inmigración se pregunta si son los partidos políticos estructuras capaces todavía de condicionar a los individuos en su forma de percibir la inmigración.

En conclusión, el pluralismo metodológico parece motivar no sólo a Barataria, sino más en general, a todas las actividades de la ACMS. Individuo y sociedad, en la mayoría de los artículos y ponencias analizados, se configuran como dos caras de una misma realidad. Ambos paradigmas, el de la acción colectiva y el individualismo metodológico, dejan demasiados problemas sin resolver (Perulli, 2015: 115) así como dejan "la imposibilidad de indicar un sentido estable del ser" (Spreafico, 2015: 55). Es evidente que resulta absurdo seguir contraponiendo lo individual y lo universal, dos modelos que en el pasado "se han ido acumulando conflictivamente" (García Selgas, 2015: 70; Camas Baena, 2014: 150). En síntesis, la pluralidad de la sociología, que se hace evidente en las actividades de la ACMS, no oscurece, sino todo lo contrario, el hecho de que el discurso sociológico es un análisis científico en el que participan, con igual dignidad, "modelos teóricos diferentes" (García Selgas, 2015: 70). De hecho, en un mundo fluido y que sufre procesos de cambios frecuentes, todas las contraposiciones binarias, que tanto espacio tuvieron en la sociología y antropología de otras épocas, entran en crisis (Beriain, 2015: 4).

\section{TEMÁTICAS ESPECIALMENTE TRATADAS POR LA REVISTA: LAS FUERZAS ARMADAS Y LOS NÚMEROS EXTRAORDINARIOS}

Nos parece haber detectado, analizando las actividades de la ACMS, que el tema de las Fuerzas Armadas es de los más tratados en artículos y ponencias de los congresos. A continuación vamos a comprobar si esta hipótesis tiene fundamento.

En casi todos los congresos la ACMS ha dedicado efectivamente una mesa y varias ponencias al tema de las Fuerzas Armadas. En el quinto número de Barataria los profesores Martínez Paricio y Díaz Castro recuerdan la importancia que tuvo el fallido golpe de estado del 23-F para cambiar las Fuerzas Armadas y solucionar "el problema militar en España" (Martínez Paricio y Díaz Castro 2002: 197). En el séptimo número de Barataria dos miembros históricos de la ACMS, los profesores Roberto Barbeito y 
Mercedes Fernández Antón (2007: 67 y ss.), analizan el papel de los museos militares en España, tomando como referencia comparativa un museo extranjero, el del Aire de Duxford. El artículo, que es el resultado de una amplia investigación basada en una triangulación metodológica, presenta un completo marco teórico sobre el concepto polisémico de cultura. También Hierro Roldán (ACMS, 2007: 499) presentó en Almagro una ponencia sobre el servicio militar obligatorio en España y su abolición.

En el undécimo número de la revista Puertas Cristóbal y Fernández Alles (2010: 121 y ss.) recuerdan que las Fuerzas Armadas no cumplen sólo acciones militares sino también una importante función social y humanitaria. En el XIII Congreso, María Gómez Escarda (ACMS, 2008: 279 y ss.) presenta la evolución de las Fuerzas Armadas en relación al cambio social. La autora analiza los cambios que se han producido desde la llegada de la profesionalización del ejército y la supresión del servicio militar obligatorio. Se llega así a demostrar que en el Ejército ha pasado lo mismo que en la sociedad española en su globalidad: aumento del número de extranjeros, del número de mujeres (del 0,1\% en 1991 al 12,3\% en 2007) (ACMS, 2008: 287), y pluralidad familiar. Además se ha producido un aumento del número de matrimonios entre hombres y mujeres militares.

La última parte de este recorrido por los contenidos de Barataria va dedicado a los números monográficos y cuyas aportaciones, como su nombre indica, van destinadas a temáticas concretas. Así tenemos los números 13 y 15, coordinados por el profesor Manuel Canales. El número 13 está dedicado a las políticas migratorias y en él se recogen artículos sobre los efectos, problemática y retos que plantea este hecho en las sociedades postmodernas actuales. En el número 15, los artículos nos hablan de las políticas públicas sociales en tiempos de crisis, dada su gran actualidad e incidencia social tal y como lo reflejan tanto los medios de comunicación como las encuestas de opinión.

Los números 17 y 19, coordinados por el profesor Alfonso Ortega, nos hablan sobre inmigración, nacionalidad y extranjería, con artículos de relevante actualidad que tratan de dar respuesta a los retos, a veces problemáticos, que estos hechos plantean en nuestra sociedad a comienzos del siglo XXI, que no van a cejar a corto plazo y que se deben afrontar con un alto grado de valentía para hacer frente a las realidades a ambos lados de la frontera, sea esta cual sea.

\section{CONCLUSIONES}

La ACMS ha hecho un gran trabajo por desarrollar la sociología en Castilla-La Mancha. Podemos perfilar, no obstante, a partir del análisis de los artículos y ponencias que hemos llevado a cabo, que la sociología manchega necesitaría un estudio monográfico sobre su origen, su historia y sus direcciones actuales, algo parecido a lo que han hecho ÁlvarezUría y Varela (2000) y Salustiano del Campo (2001) con la sociología española en general. Estamos seguros de que la ACMS hará suya pronto esta invitación o sugerencia.

\section{BIBLIOGRAFÍA}

ACMS (2007). XII Congreso de Sociología en Castilla-La Mancha. Castilla-La Mancha: 25 años de autonomía. Ponencias y Comunicaciones. Toledo: ACMS.

ACMS (2008). XIII Congreso de Sociología en Castilla-La Mancha. Sociedad, consumo y sostenibilidad. Toledo: ACMS. 
ACMS (2009). XIV Congreso de Sociología en Castilla-La Mancha. Igualdad, desarrollo y cooperación. Toledo: ACMS.

ACMS (2010). XV Congreso de Sociología en Castilla-La Mancha. Construcciones y deconstrucciones de la sociedad. Toledo: ACMS.

ÁLVAREZ-URÍA, F. y VARELA, J. (2000). La galaxia sociológica. Madrid: La Piqueta.

BENITO GIL, V. J. (2012). "Crisis, globalidad y migraciones: perspectivas de los nuevos tiempos", en Barataria, 13: 31-46.

BERIAIN, J. (2015). "Genealogía afirmativa del hecho religioso en perspectiva sociológica", en REIS, 151:3-22.

BLEDA GARCÍA, J. M. (2002). El Estado de Bienestar en la Comunidad de Castilla-La Mancha. Toledo: Ediciones Parlamentarias de Castilla-La Mancha.

-(2010). "Felipe Centelles, un soñador de la sociología que construyó realidades", en Barataria, 11: 15-17.

BRASTER, J.F.A., DEL POZO A. y DEL MAR, M. "Características y tendencias de la Educación Primaria en Castilla-La Mancha (1900-1975)", en A. Del Pozo A. y M. Del Mar (coords.) (2006). La educación en Castilla-La Mancha en el siglo XX (1900-1975). Ciudad Real: Almud.

CALLEJO, J. (2007). "Estructuras de empleo del tiempo en la Comunidad de Castilla-La Mancha", en Barataria, 8: 143-159.

CAMAS BAENA, V. (2014). "La mirada etnobiográfica como espacio interdisciplinar en la investigación social", en Methaodos, 2: 148-170.

CANO HILA, A. B. (2014). "Jóvenes, expectativas de futuro y desempleo juvenil. ¿La condición de nini-ni explicaba realmente el desempleo juvenil en España en tiempo de bonanza económica?", en ACMS (2014). XII premios de ensayo breve en Ciencias Sociales. Toledo: ACMS, pp. 12-33.

CENTELLES BOLÓS, F. (2008). "Percepción de Castilla-La Mancha según los universitarios", en F. Centelles Bolós (ed.) Castilla-La Mancha: la consolidación de un proyecto social. Veinticinco años de autonomía. Cuenca: Ediciones de la Universidad de Castilla-La Mancha, pp. 33-55.

- y GARCÍA LÓPEZ, J. (2004). "Evolución socioeconómica de Castilla-La Mancha (año 1990)", en Praxis Sociológica, 8: 37-46.

CLEMENTE, M., ESPINOSA, P. y FERNÁNDEZ ANTÓN, M. (2013). "La integración de los inmigrantes a través de la comunicación", en Barataria, 16: 83-96.

DE LA CALLE VALVERDE, J. (2011). "La tesis doctoral: el rito sin paso", en ACMS. IX Premio de Ensayo Breve en Ciencias Sociales Fermín Caballero. Toledo: ACMS, pp. 9-29.

DEL CAMPO, S. (2001). Historia de la sociología española. Ariel: Barcelona.

DE MIGUEL CALVO, E. (2011). "Emociones y desigualdades sociales. El caso del miedo", en ACMS, IX Premio de Ensayo Breve en Ciencias Sociales Fermín Caballero. Toledo: ACMS, pp. 49-75.

DÍAZ CANO, E. (2007). "Ernst Troeltsch: una aproximación", en ACMS, XII Congreso Nacional de Sociología en Castilla-La Mancha. Toledo: ACMS, pp. 27-42.

DUMONT, G. y CLUA GARCÍA, R. (2015). "Acercamiento socio-antropológico al concepto de estilo de vida", en Aposta, 66: 83-99.

DURÁN HERAS, M.A. (2001). "La actual institucionalización de la sociología en España”, en S. Del Campo (dir.) (2001). Historia de la sociología española. Barcelona: Ariel, pp. 285-321.

ENCUESTA CONDICIONES DE VIDA (2014). En www.ine.es [consulta 26/5/2015].

EPA 2015 (2015). En www.ine.es [consulta 25/4/2015].

EUROSTAT (2015). Clasificación de las regiones por PIB per cápita en paridad de poder de compra, en http://ec.europa.eu/eurostat/help/new-eurostat-website [consulta 1/6/2015].

FERNÁNDEZ ANTÓN, M. y BARBEITO IGLESIAS, R.L. (2005-2006). "El papel de los museos militares en el fomento de una "cultura de la defensa" democrática", en Barataria, 7: 63-89.

FERNÁNDEZ FERNÁNDEZ, M. (2010). "Tradiciones y estudios actuales de Sociología de la comunicación", en Barataria, 10: 139-157.

FRANCO DE MACEDO, R. (2010). "Sobre la actualidad del pensamiento de Francisco de Vitoria en la sociedad globalizada del siglo XXI", en Barataria, 11: 41-56.

FUNDACIÓN ENCUENTRO (2013). Informe España 2013. Una interpretación de su realidad social, en www.fund-encuentro.org [consulta 30/7/2015].

FUNDACIÓN FOESSA (2014). VII Informe: exclusión y desarrollo social en España. Madrid: Foessa. 
FUNDACIÓN 1 DE MAYO (2013). Crisis económica y nuevo panorama migratorio en España, Centro de Documentación de las migraciones de la Fundación $1^{\circ}$ mayo, en www.1mayo.ccoo.es [consulta 1/5/2015].

GALLEGO TRIJUEQUE, S. (2011). "Redes sociales y desarrollo humano", en Barataria, 12: 113-122. GARCÍA BILBAO, P.A. (2012). "Migraciones forzosas en el mundo clásico: Plutarco y la construcción social de la realidad", en Barataria, 14: 157-170.

GARCÍA MANSO, A. (2007). "Cyborg, mujeres y debates. El ciberfeminismo como teoría y crítica", en Barataria, 8: 13-26.

GARCÍA SELGAS, F.J. (2015). "Tres modelos teóricos generales en sociología: una des-unidad articulada", en REIS, 151: 65-82.

GONZÁLEZ-ANLEO, J.M. (2014). Consumidores consumidos. Juventud y cultura consumista. Madrid: Khaf.

HORMIGOS RUIZ, J. (2012). "La sociología de la música. Teorías clásicas y puntos de partida en la definición de la disciplina", en Barataria, 14: 75-84.

INE (2014). Anuario Estadístico de España 2014, en www.ine.es [consulta 25/4/2015].

INE (2014). Anuario Estadístico de España. Cultura y ocio, en www.ine.es [consulta 8/6/2015].

INGELLIS, A. J. y CALVO, R. (2015). "Desempleo y crisis económica. Los casos de España e Italia", en Sociología del Trabajo, 84: 7-31.

LÓPEZ ÁLVAREZ, A. (2014). "La inmigración y los partidos políticos en España", en Barataria, 17: 203-213.

LÓPEZ LÓPEZ, P. y ESCALADA LÓPEZ, C. (1999). "Años de sociología española a través de la Revista Española de Investigaciones sociológicas (1978-1997)", en Revista General de Información y Documentación, vol.9, 1: 161-173.

MANTOVANI, G. (2008). Analisi del discorso e contesto sociale. Bologna: Il Mulino.

MARTÍN CABELLO, A. (2002). "Sociología de la cultura y sociología cultural" en Barataria, 5: 173179.

MARTÍN CRIADO, E. (2014). “Mentiras, inconsistencias y ambivalencias”, en RIS, 72 (1): 115-138.

MARTÍN CRIADO, E. (1998). Producir la juventud. Madrid: Istmo.

MARTÍNEZ DE LIZARRONDO ARTOLA, A. (2009). "La senda hacia una política transversal de integración de inmigrantes en Castilla-La Mancha", en Barataria, 10: 75-90.

MARTÍNEZ PARICIO, J.I. y DÍAZ CASTRO, J.R. (2002): "Las fuerzas armadas en el nuevo ciclo de la vida política española”, en Barataria, 5: 195-212.

MOLINA, J. (2000): “Conflicto, política y polemología en el pensamiento de Julien Freund”, en Barataria, 2-3: 177-217.

MUÑOZ SÁNCHEZ, E. (2006). Mujer y educación en Castilla-La Mancha durante el siglo XX. De la liberación a la nacionalización", en M del M. Del Pozo Andrés (coord.) La educación en Castilla-La Mancha en el siglo XX. Ciudad Real: Almud, pp. 357-384.

NOGUERA, J.A. (2003). “¿Quién teme al individualismo metodológico?”, en Papers, 69: 101-132.

NOYA, J. (2004). "Teoría e investigación en la sociología española contemporánea", en Política y Sociedad, vol.41, 2: 163-174.

NUÑEZ SÁNCHEZ, E.M. (2013). "Comunitat Valenciana: una integración de vanguardia "la Escuela de acogida", en Barataria, 13: 75-86.

PASCALE, C.M. (2010). "Horizontes de posibilidad: sociología para el siglo XXI", en Barataria, 11: 31-39.

PERELLÓ OLIVER, S. (2009). Metodología de la investigación social, Madrid, Dykinson.

PÉREZ YRUELA, M. (2011). "La sociología hace treinta años y la sociología dentro de treinta años", en RES, 15: 135-146.

PERULLI, P. (2015). “Crisi e paradigmi delle scienze sociali”, en Rassegna Italiana di Sociologia, 1: 97-121.

PUERTAS CRISTÓBAL, E. y FERNÁNDEZ ALLES, J.J. (2010). "El papel de las fuerzas armadas en la acción humanitaria", en Barataria, 11: 121-138.

ROMANIA, V. (2009). "L'immigrato e il malinteso della sicurezza: L'influenza del frame sicuritario nella sperimentazione di una pratica innovativa di mediazione culturale su strada", Barataria, 10: 39-55. 
SANTIAGO GARCÍA, J.A. (2015). "La estructura social a la luz de las nuevas sociologías del individuo", en REIS, 149: 131-150.

SPREAFICO, A. (2015). "Decostruzioni e categorizzazioni: una questione rilevante per una etnometodologia critica", en Rassegna Italiana di Sociologia, 1: 49-74.

SUÁREZ ÁVILA, P.V. (2012). "Activismo político de la sociedad civil migrante y gobierno local: la política pública migratoria en San Francisco", en Barataria, 13: 195-209.

UÑA JUÁREZ, O. (1998). "Cuestiones epistemológicas específicas de las ciencias sociales", en Barataria, 1: 11-63.

- (2000). "Prólogo", en J.M. Bleda García, F. Centelles Bolós y F. Mora (2000). Construcción de la identidad política (Castilla-La Mancha). Olías del Rey: Azacanes, pp. 5-7.

- (2005-2006). "La constitución de la sociología como ciencia", en Barataria, 7: 91-116.

- (2008). "Presentación”, en F. Centelles Bolós (ed.) Castilla-La Mancha: la consolidación de un proyecto social. Veinticinco años de autonomía. Cuenca: Ediciones de Universidad de Castilla-La Mancha, pp. 13-14.

-, BLEDA GARCÍA, J. M. y CENTELLES BOLÓS, F. (1999). La mujer en Castilla-La Mancha. Un estudio sociológico. Cuenca: Ediciones de la Universidad de Castilla-La Mancha.

-, HORMIGOS RUIZ, J. y MARTÍN CABELLO, A. (coords.) (2007). Las dimensiones sociales de la globalización. Madrid: Paraninfo.

UNICEF (2010). Abandono escolar temprano, en www.unicef.es [consulta 11/6/2015].

URTEAGA, E. (2013). "El pensamiento de Norbert Elias: proceso de socialización y configuración social", en Barataria, 16: 15-31.

VALLES, M.S. (1999). Técnicas cualitativas de investigación social. Madrid: Síntesis.

VERA, H. (2012). "Por una sociología histórica del conocimiento", en Sociología Histórica, 1: 239-250.

\section{Breve currículo:}

\section{Giuliano Tardivo}

Doctor cum laude por la Universidad Rey Juan Carlos (Madrid), licenciado en Ciencias Políticas por la Universidad de Padua (Italia), título homologado por la Universidad Complutense. Máster en Instituciones locales e integración europea y en Gestión e Investigación de la Comunicación empresarial. Desde 2009 es profesor en la URJC. Becario predoctoral desde 2005 hasta 2009. 